\title{
Charmed Meson Production Deep Inelastic Scattering at HERA and Extraction of $F_{2}^{c \bar{c}}$
}

\author{
Karin Daum ${ }^{* i}$ \\ Bergische Universität Wuppertal, Gaußstraße 20, D-42097 Wuppertal, Germany \\ E-mail: daumemail.desy.de
}

\section{On behalf of the $\mathrm{H} 1$ and ZEUS collaborations}

Recent results from the H1 and ZEUS collaborations on charmed meson production in deep inelastic ep scattering at HERA are presented. Single differential cross sections of $D^{* \pm}, D^{ \pm}$and $D^{0}$ meson production are measured in the kinematic range $5 \mathrm{GeV}^{2}<Q^{2}<1000 \mathrm{GeV}^{2}$ and $0.02<y<0.7$. Leading and next-to-leading order QCD predictions are compared to these measurements. The charm contribution $F_{2}^{c \bar{c}}$ to the proton structure function is derived by extrapolating the visible cross sections to full phase space.

European Physical Society Europhysics Conference on High Energy Physics, EPS-HEP 2009, July 16 - 222009

Krakow, Poland

\footnotetext{
* Speaker.

†Permanent address: DESY, Notkestarße 85, D-22603 Hamburg, Germany
} 


\section{Introduction}

The measurement of the inclusive charm quark cross sections in deep inelastic ep scattering at HERA is an important test of quantum chromodynamics $(Q C D)$. At leading order $(L O)$ charm quarks are predominantly produced by the photon gluon fusion $(P G F)$ process, i.e. $\gamma g \rightarrow c \bar{c}$, where a photon emitted by the electron interacts with a gluon in the proton. The process $e p \rightarrow e D X$ is therefore directly sensitive to the gluon density in the proton which is of utmost importance for understanding the physics to be expected at the LHC [1].

The mass $m_{c}$ of the charm quark implies a sufficiently high scale to apply perturbation theory for calculating this process in QCD without any phase space restrictions. However, there are additional scales involved, e.g. the virtuality $Q^{2}$ of the exchanged photon and the transverse momenta $p_{\perp}$ of the outgoing quarks which makes this process a multi-scale problem. Depending on the relative magnitude of $m_{c}, Q$ and $p_{\perp}$ different approaches in $\mathrm{pQCD}$ have been elaborated.

At low values of $Q, p_{\perp} \approx \mathscr{O}\left(m_{c}\right)$ the massive fixed flavour number scheme $(F F N S)$ is applicable [2]. Heavy quarks are generated dynamically via the PGF process, they are not part of the proton's parton density functions $(P D F)$. Different next-to-leading order $(N L O)$ programs were developed in this scheme for different purposes, i.e. the Riemersma program [3] for calculating the charm contribution $F_{2}^{c \bar{c}}$ to the proton structure and the HVQDIS [4] program providing parton level kinematics for calculating exclusive processes like $D$ meson cross sections.

At high scales when $Q, p_{\perp} \gg m_{c}$ the zero mass variable flavour number scheme (ZM-VFNS) is appropriate [5], where the heavy quark masses are neglected and charm and beauty becomes part of the proton PDFs.

More recently, calculations in the general mass variable flavour number scheme (GM-VFNS) became available [6] which incorporates the two approaches described above in their appropriate phase space regimes and interpolates between both schemes in a transition region.

\section{Inclusive $D^{* \pm}$ meson production}

This analysis [7] is based on an integrated luminosity of $\mathscr{L}_{\text {int }}=347 \mathrm{pb}^{-1}$ recorded by the $\mathrm{H} 1$ experiment in the year 2004-2007 where either electrons or positrons with an energy of $27.6 \mathrm{GeV}$ were collided with protons of $920 \mathrm{GeV}$. $D^{* \pm}$ mesons are identified by the decay chain $D^{* \pm} \rightarrow$ $D^{0} \pi^{ \pm}, D^{0} \rightarrow K^{\mp} \pi^{ \pm}$in the range $p_{\perp}\left(D^{*}\right)>1.5 \mathrm{GeV}$ and $\left|\eta\left(D^{*}\right)\right|<1.5$ in the laboratory frame. DIS events were selected in the kinematic region $0.02<y<0.7$ and $5 \mathrm{GeV}^{2}<Q^{2}<$ $1000 \mathrm{GeV}^{2}$.

Figure 1 shows the inclusive differential $D^{* \pm}$ cross sections as a function of $p_{\perp}$ separately for $Q^{2}<100 \mathrm{GeV}^{2}$ and for $Q^{2}>100 \mathrm{GeV}^{2}$ and as a function of $Q^{2}$. It also includes the NLO FFNS predictions from HVQDIS using the proton PDFs CTEQ5F3[8] and MRST2004FF3nlo[9]. The bands indicate the theoretical uncertainties due to variation of the fragmentation parameters, the charm quark mass $m_{c}$ and the scales $\mu_{r}=\mu_{f}$. These calculations agree reasonably well with data albeit there are differences in shape in the $p_{\perp}$ distribution at low $Q^{2}$ and in the $Q^{2}$ distribution. The predictions from the LO+PS Monte Carlo programs Rapgap [10] and Cascade [11] which are compared to the measurement of $\mathrm{d} \sigma / \mathrm{d} p_{\perp}$ at high $Q^{2}$ fail to describe the data. 

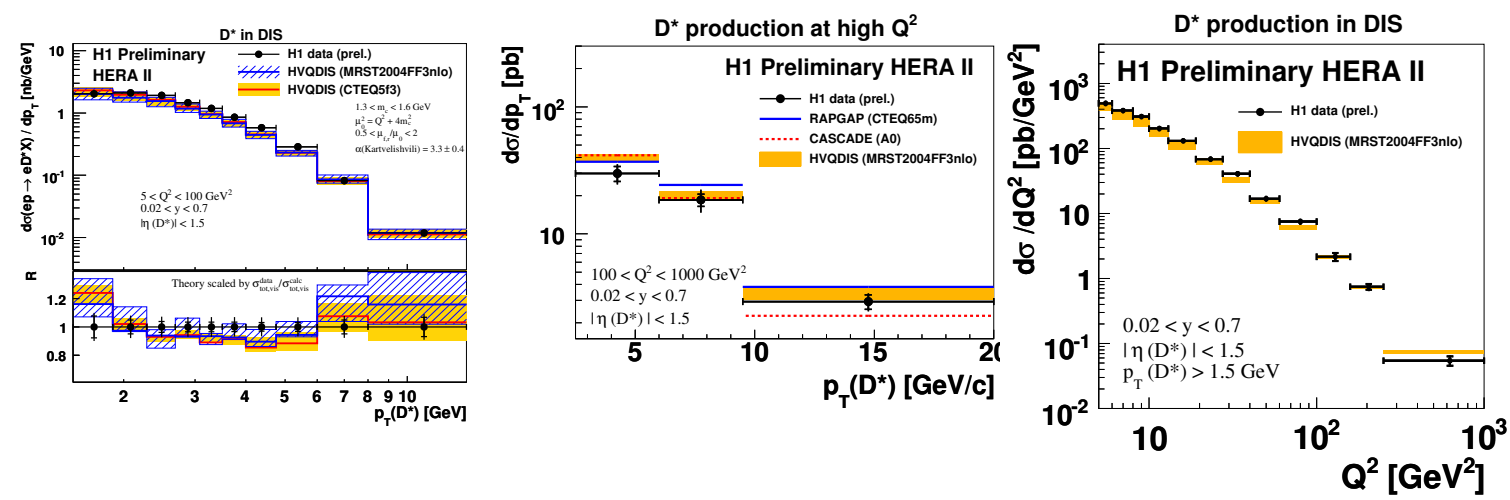

Figure 1: Differential $D^{* \pm}$ cross sections as function of $p_{\perp}$ for $5<Q^{2}<100 \mathrm{GeV}^{2}$ (left) and for $100<Q^{2}<1000 \mathrm{GeV}^{2}$ (centre) and as a function of $Q^{2}$ (right).

\section{Inclusive $D^{0}$ and $D^{ \pm}$meson production}

The ZEUS collaboration has analyzed the inclusive $D^{0} / D^{\overline{0}}$ and $D^{ \pm}$production in DIS [12] based on an integrated luminosity of $\mathscr{L}_{\text {int }}=133.6 \mathrm{pb}^{-1}$ collected in the years 2004 and 2005 . The $D$ mesons are identified via the decay modes $D^{0} / D^{\overline{0}} \rightarrow K^{\mp} \pi^{ \pm}$and $D^{ \pm} \rightarrow K^{\mp} \pi^{ \pm} \pi^{ \pm}$. The decay products of a $D$ meson candidate are required to originate from a common secondary vertex reconstructed in the micro-vertex detector well separated from the primary vertex. Charm meson cross sections for the process $e p \rightarrow e D X$ were calculated using the reconstructed $D^{+}$and $D^{0}$ mesons in the kinematic region $5 \mathrm{GeV}^{2}<Q^{2}<1000 \mathrm{GeV}^{2}, 0.02<y<0.7,1.5 \mathrm{GeV}<p_{\perp}(D)<15 \mathrm{GeV}$ and $|\eta(D)|<1.6$.

Figure 2 shows the inclusive differential $D^{ \pm}$and $D^{0} / D^{\overline{0}}$ cross sections as a function of $p_{\perp}$ and $Q^{2}$ together with the expectation from the NLO FFNS program HVQDIS using the proton PDF ZEUS-S NLO obtained from a fit [13] to the inclusive $F_{2}$ measurements. The total uncertainties on this prediction due to the uncertainties on the ZEUS PDF, the charm quark mass, the scales $\mu_{r}=\mu_{f}$ and the fragmentation function are indicated by the blue bands. The NLO FFNS calculations agree well with the data, both in $D^{ \pm}$and $D^{0} / D^{\overline{0}}$ production.
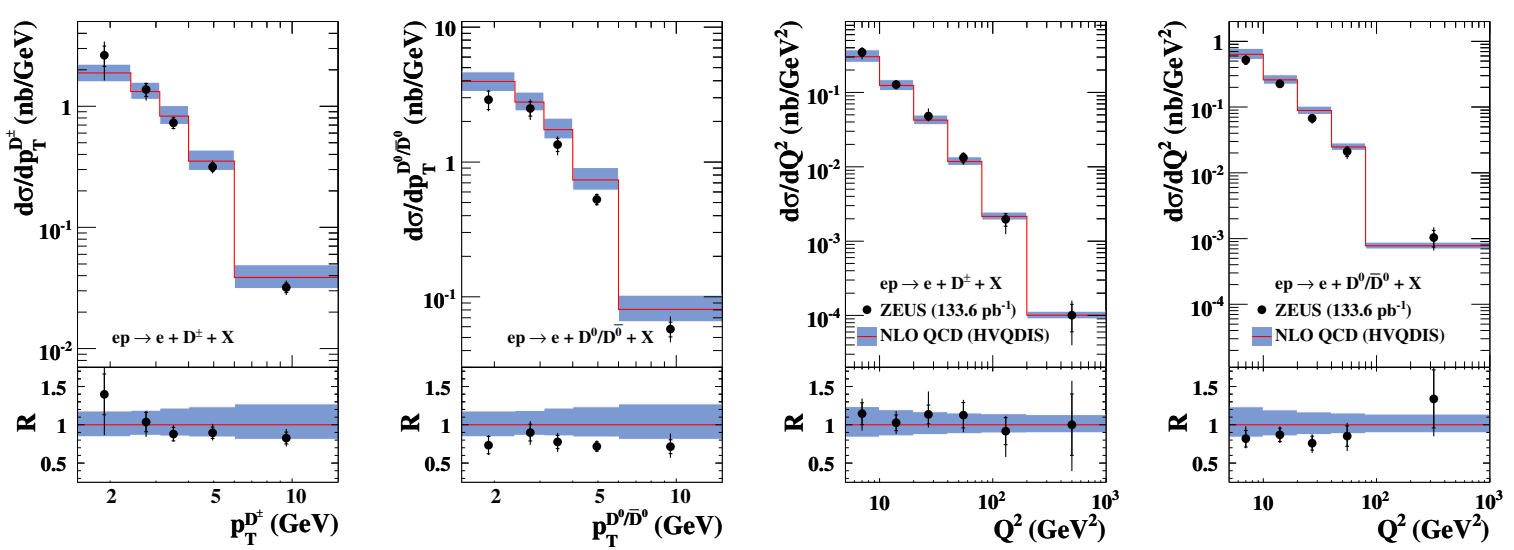

Figure 2: Differential $D^{ \pm}$and $D^{0} / D^{\overline{0}}$ cross sections as function of $p_{\perp}$ (left) and as a function of $Q^{2}$ (right). 

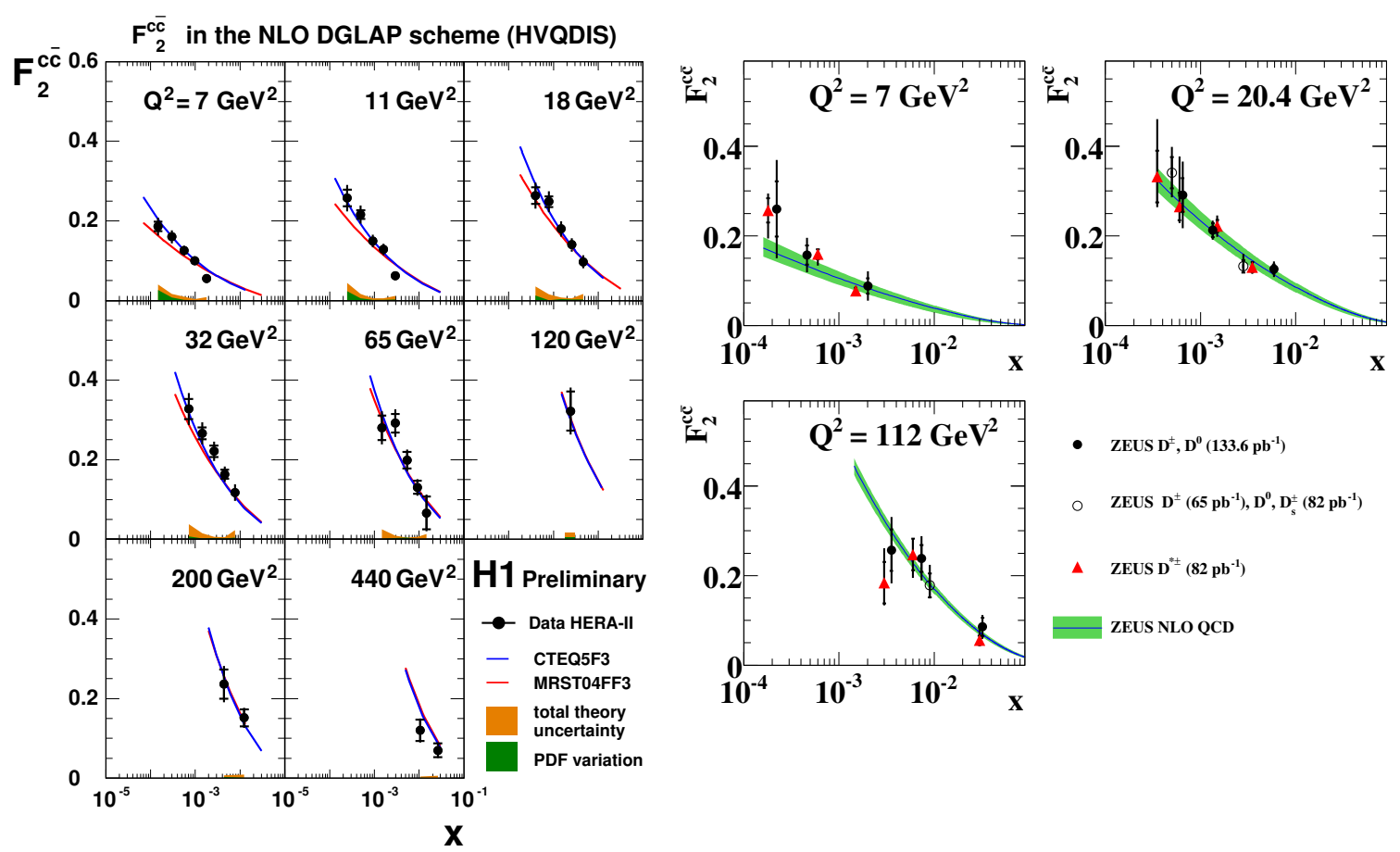

$\Delta \quad \operatorname{ZEUS~D}^{* \pm}\left(\mathbf{8 2} \mathbf{p b}^{-1}\right)$

ZEUS NLO QCD

Figure 3: $F_{2}^{c \bar{c}}$ obtained from the inclusive $D$ cross section measurements by H1 (left) and ZEUS (right). The inner(outer) error bars refer to the statistical (total) uncertainties, respectively. The curves shows the predictions from NLO pQCD in the FFNS scheme based on the CTEQ5F3(blue), MRST04FF3(red) and ZEUS(green) proton PDFs.

\section{Charm contribution $F_{2}^{c \bar{c}}\left(x, Q^{2}\right)$ to the proton PDF}

The charm contribution, $F_{2}^{c \bar{c}}$, to the inclusive proton structure function $F_{2}$ is obtained using the expression for the one photon exchange cross section for charm production

$$
\frac{\mathrm{d}^{2} \sigma^{c \bar{c}}}{\mathrm{~d} x \mathrm{~d} Q^{2}}=\frac{2 \pi \alpha_{e m}^{2}}{Q^{4} x}\left(\left[1+(1-y)^{2}\right] F_{2}^{c \bar{c}}\left(x, Q^{2}\right)-y^{2} F_{L}^{c \bar{c}}\left(x, Q^{2}\right)\right),
$$

where the contribution from the longitudinal structure function $F_{L}^{c \bar{c}}$ is accounted for and amounts to at most $4 \%$ in the $Q^{2}-y$ plane explored at HERA. The measured visible $D$ meson cross sections $\sigma_{v i s}^{e x p}\left(x, Q^{2}\right)$ in bins of $x$ and $Q^{2}$ are converted to a bin centre corrected values of $F_{2}^{c \bar{c}}\left(\langle x\rangle,\left\langle Q^{2}\right\rangle\right)$ according to

$$
F_{2}^{c \bar{c}}\left(\langle x\rangle,\left\langle Q^{2}\right\rangle\right)=\frac{\sigma_{v i s}^{e x p}\left(x, Q^{2}\right)}{\sigma_{v i s}^{\text {theo }}\left(x, Q^{2}\right)} \cdot F_{2}^{c \bar{c} \text { theo }}\left(\langle x\rangle,\left\langle Q^{2}\right\rangle\right),
$$

where $\sigma_{v i s}^{\text {theo }}$ and $F_{2}^{c \bar{c}}$ theo are the theoretical predictions of the NLO FFNS calculations.

Figure 3 shows the measurements of $F_{2}^{c \bar{c}}$ extracted from the inclusive cross sections of $D^{* \pm}$ (left) and of $D^{0}$ and $D^{ \pm}$(right) meson production from H1 [14] and ZEUS [12], respectively. The data are compared to the massive FFNS NLO calculation [3] based on the proton PDFs from global analyses MRST04FF3nlo[9] (red), CTEQ5F3[8] (blue) and ZEUS-S NLO QCD fit[13] (green band) to inclusive $F_{2}$ data. The bands in both panels indicate the theoretical uncertainties due to parameter variations in the calculations as described in section 2 and 3 with the exception of the fragmentation uncertainty, which does not enter here. The theoretical predictions from the global analyses are found to agree reasonably well with the $F_{2}^{c \bar{c}}$ measurements. 


\section{Conclusions}

New results from the H1 and ZEUS experiments on $D$ meson production in DIS have been presented. The differential inclusive cross sections are found to be well described by the NLO FFNS calculations. By extrapolating the $D$ meson production cross sections from the visible to the full phase space the charm contribution $F_{2}^{c \bar{c}}$ has been determined. The NLO FFNS predictions using proton PDFs obtained by global analyses yield reasonable descriptions of the experimental data.

\section{References}

[1] R. S. Thorne and W. K. Tung, Proceedings of the HERA and the LHC workshop series on the implications of HERA for LHC physics (2009) 332 ISBN 978-3-935702032-4; [arXiv:0809.0714].

[2] E. Laenen et al., Phys. Lett. B291 (1992) 325.

[3] E. Laenen et al., Nucl. Phys. B392 (1993) 162, ibid. 229; S. Riemersma, J. Smith, and W.L. van Neerven, Phys. Lett. B347 (1995) 143.

[4] B.W. Harris and J. Smith, Nucl. Phys. B452 (1995) 109; Phys. Lett. B353 (1995) 535; Phys. Rev. D57 (1998) 2806.

[5] B.A. Kniehl et al., Z. Phys. C76 689 (1997); M. Cacciari and M. Greco, Phys. Rev. D55 7134 (1997); J. Binnewies et al., Z. Phys. C76 677 (1997); J. Binnewies et al.), Phys. Rev. D58 014014 (1998).

[6] M.A.G. Aivazis et al., Phys. Rev. D50 (1994) 3102; R.S. Thorne and R. G. Roberts, Phys. Rev. D57 (1998) 6871; P. M. Nadolsky et al., Phys. Rev. D78 (2008) 013004 [arXiv:hep-ph/802.0007]; A.D. Martin et al., [arXiv:hep-ph/0901.0002].

[7] [H1 Coll.], Contributed paper to the " $34^{\text {th }}$ International Conference on High Energy Physics", ICHEP2008, Philadelphia, Abstracts 855 and 860.

[8] H.L. Lai et al., [CTEQ Coll.], Eur. Phys. J. C12 (2000) 375, hep-ph/9903282.

[9] A.D. Martin, W.J. Stirlin and R.S. Thorne, Phys Lett. B636 (2006) 259, hep-ph/0603143.

[10] H. Jung, Comp. Phys. Commun.86 (1995) 147.0

[11] H. Jung, Proc. 7th DIS Workshop, Nucl. Phys. (Proc. Suppl.) B79 (1999) 429.

[12] S. Chekanov et al. [ZEUS Coll.],Eur. Phys. J. C 10.1140/epjc/s10052-009-1088-x.

[13] S. Chekanov et al. [ZEUS Coll.], Phys. Rev. D67 (2003) 12007.

[14] [H1 Coll.], Contributed paper to the " $34^{\text {th }}$ International Conference on High Energy Physics", ICHEP2008, Philadelphia, Abstract 867. 